\title{
A Study on Computerized Simulation of difference between CPM and CCPM
}

\author{
Zhang Fan \\ School of Architecture and Engineering, Yan'an University, Yan'an 716000, China \\ 121604203@qq.com
}

Keyword: computerized simulation, CCPM+, CPM, CCPM

\begin{abstract}
The constant delays of projects demonstrate that traditional project planning methods may adopt an inefficient way to manage uncertainty. To tackle problems in traditional project system, CCPM (Critical Chain Project Management) was introduced by Goldratt(1997) to identify both task dependence and resource conflicts as main constraints within a project[1].This method is characterized by applying Theory of Constraints in project management and inserting buffers to deal with uncertainty. Although CCPM have demonstrated certain success and is regarded as an alternative of traditional project planning technique, lots of projects still suffer from considerable failure.Given the condition that CCPM is still under doubt and has not been applied widely,this paper conducts investigation about difference between CCPM and CPM/PERT based on computerized simulation of case study.
\end{abstract}

\section{Introduction}

Projects usually fail to achieve the objectives and constantly deliver late, overrun budget or compromise the scope. To discover problems inherent in project planning, traditional planning tools including Gantt Chart, Critical Path Method (CPM) and Project Evaluation and Review Technique (PERT) have been reviewed and evaluated by lots of researchers. Despite the fact that they all have merits, they do possess a main limitation of ignoring resource limitations and only PERT addresses uncertainty.[2]Meanwhile, some other significant problems inherent in traditional project techniques includeexcessive safety duration added are reflected in the phenomenon like multitasking, student syndrome and path merging effect. [3]Therefore, the current project environment desperately needs a resource constrained planning method that takes into account uncertainty and is not too complex to manage.The followingsimulation would demonstrate the finding and discover further on a project-scheduling example.

\section{Case Study Based on Computerized simulation}

In order to achieve research purpose, an example adapted from Schieman (2002) has been used[4]. There are two projects taking place simultaneously: one is the kitchen project, and the other is the bedroom project. Microsoft Project is used to first simulate the process of CPM based on this case. The result presented in Gantt Chart could be found in Figure 1.1 (before resource leveling) and Figure 1.2( afterresourceleveling).

Then, software CCPM+, as a add-on of Microsoft Project would help to create a Critical Chain schedule after following CCPM schedulingprocedure, including:

1. Define tasks and select the MS Project schedule options.

2.Build task network.

3.Resource level the network.

4.Identify the Critical Chain.

5.Insert Feeding and Project Buffers.[5]

The result of CCPM+ simulation could be seen in Figure 1.3. 
Figure 1.1 CPM used (before resource leveling)

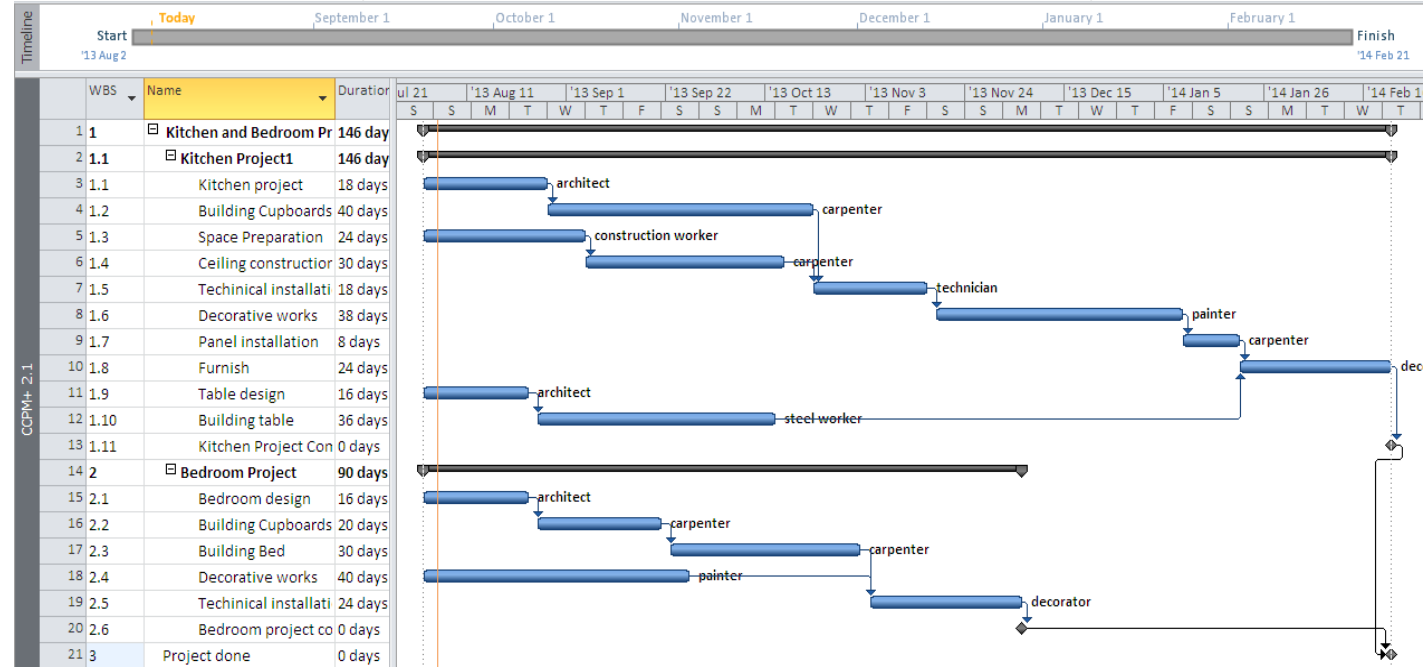

Figure 1.2 CPM used (after resource leveling)

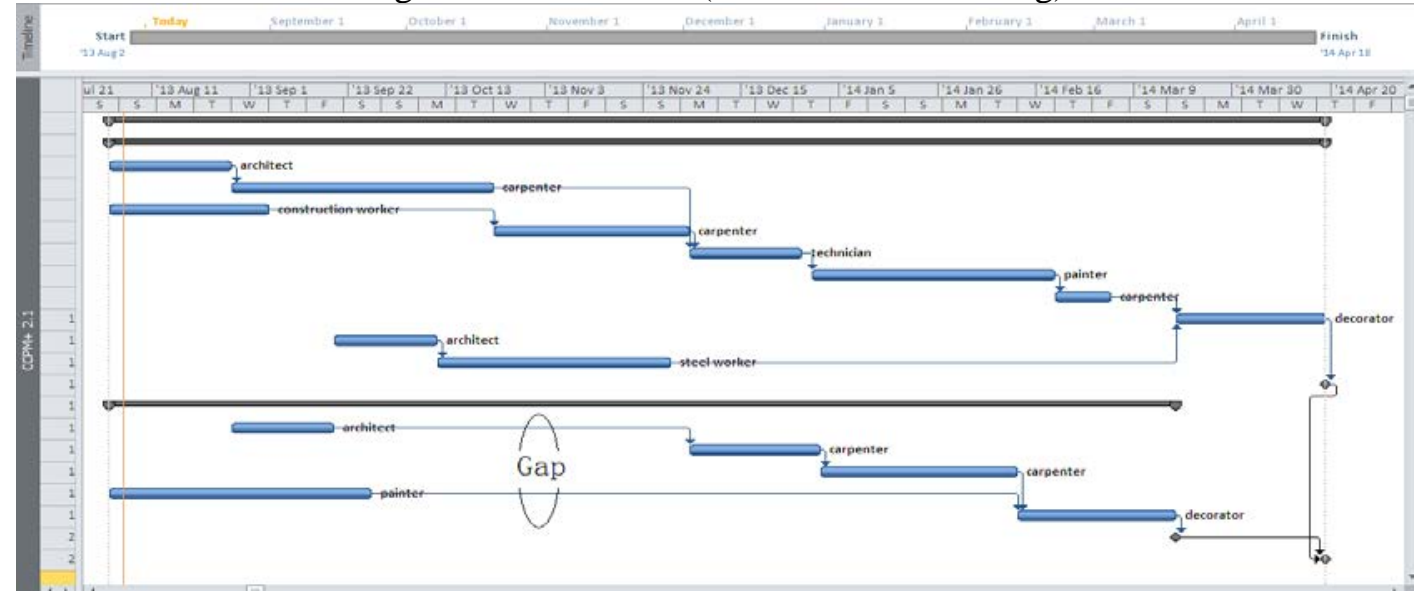

Figure 1.3 CCPM used in Kitchen and Bedroom Project

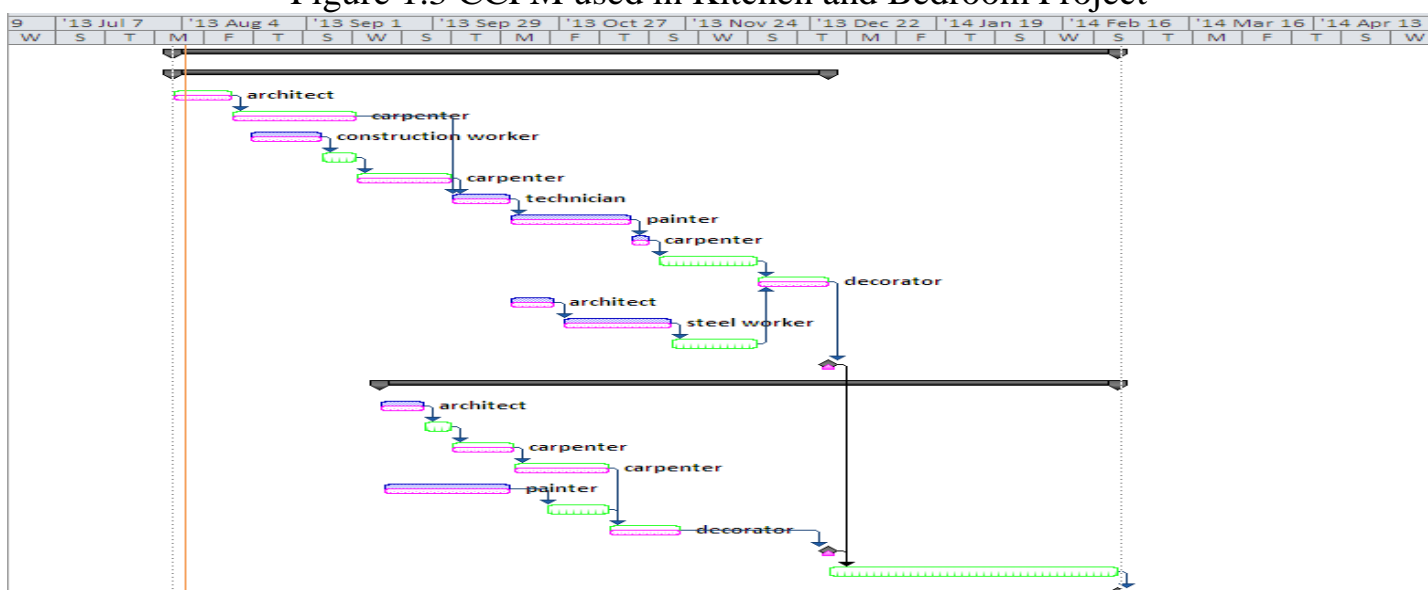

\section{Data and analysis}

According the result of above simulation, duration using CCPM (150 daysin Figure 1.3) is indeed shorter than the CPM (186 daysin Figure 1.2). In this example, it reduces the duration of 36 days. Also, it could be reflected by the extended duration of 40 days from 146 days to the leveled duration of 186 days through CPM. By contrast, CCPM enables the duration to expand only from 146 days to 150 days to handle the resource and logical constraints. Still, it is worth to mention that the chance of finishing CC project on time is larger than the CPM project. Also, this method enables the bedroom project to start as late as possible and reduce multitasking.

Further difference could be reflected from the two figures below for comparing the arrangement 
of sequence in the example project. They are summarized as follows:

1. The comparison between "late start" and "early start". In Critical Chain Method, the activities are arranged tighter than those in CPM (by comparing figure 1.1 and 1.3). "Early start" indicates that all the noncritical path tasks are allowed to start earlier than necessary to meet the schedule date. [6]However, individuals may already know there is slack available, which directly influences urgency they feel and encourage the student syndrome. In comparison,CCPM adopt "late start" for all task tasks, while adding buffers explicitly to protect the overall project from lateness, which helps to ensure project schedule protection.

2. According to Leach (2000), CPM/PERT cannot guarantee to achieve the optimum schedule. By contrast, Critical Chain regards resource conflicts as a major constraint to deal with and try to achieve the optimum [6]. The CPM levels the resources but causes unnecessary gaps shown in Figure 1.2.Since it is possible to reduce the two-circled gaps. The CCPM method identifies the real constraints for the project and then attaches important to it. By contrast, the CPM mainly follows the logical sequence of predecessor-successor task relationships and does not give the optimum plan to deal with the resource conflicts.

\section{Conclusion and recommendation}

The main problems of CPM involve the ignorance of resources contentions and the specific (deterministic) duration with predefined starting and ending dates. The computerized case study examines and confirms the inability of traditional project planning tool to recognize a project as a network of dependent events with both task and resources dependency in traditional projects planning techniques. CCPM could serve as a solution to constant projects failure in terms of scheduling by eliminating resource conflicts and inserts buffers to prevent possible lateness, though its shortcomings still need to be discovered and tackled in further study and real projects.

\section{Reference}

[1] Goldratt, E.M., (1997). Critical Chain. New York: The North River Press.

[2] Lazaros F. (2007). Comparison of the critical chain method with other project planning tool.MSc. School of Mechanical, Aerospace \& Civil Engineering. University of Manchester

[3]Horowitz J., (1967). Critical path scheduling: management control through CPM and PERT. New York : Ronald

[4]Schieman N.(2002) “CCPM and Project Success” MSc. Dissertation. University of Maastricht.

[5]Luong and Ohsato, (2008) Fuzzy Critical Chain Method for Projects Scheduling under Resource Constraints and Uncertainty .International Journal of Project Management, 26(6): 688-698.

[6]Leach P., (2000). Critical chain project management. United Kingdom: Artech House Inc

[7]Advanced Projects, (2008). Help in CCPM+ software 